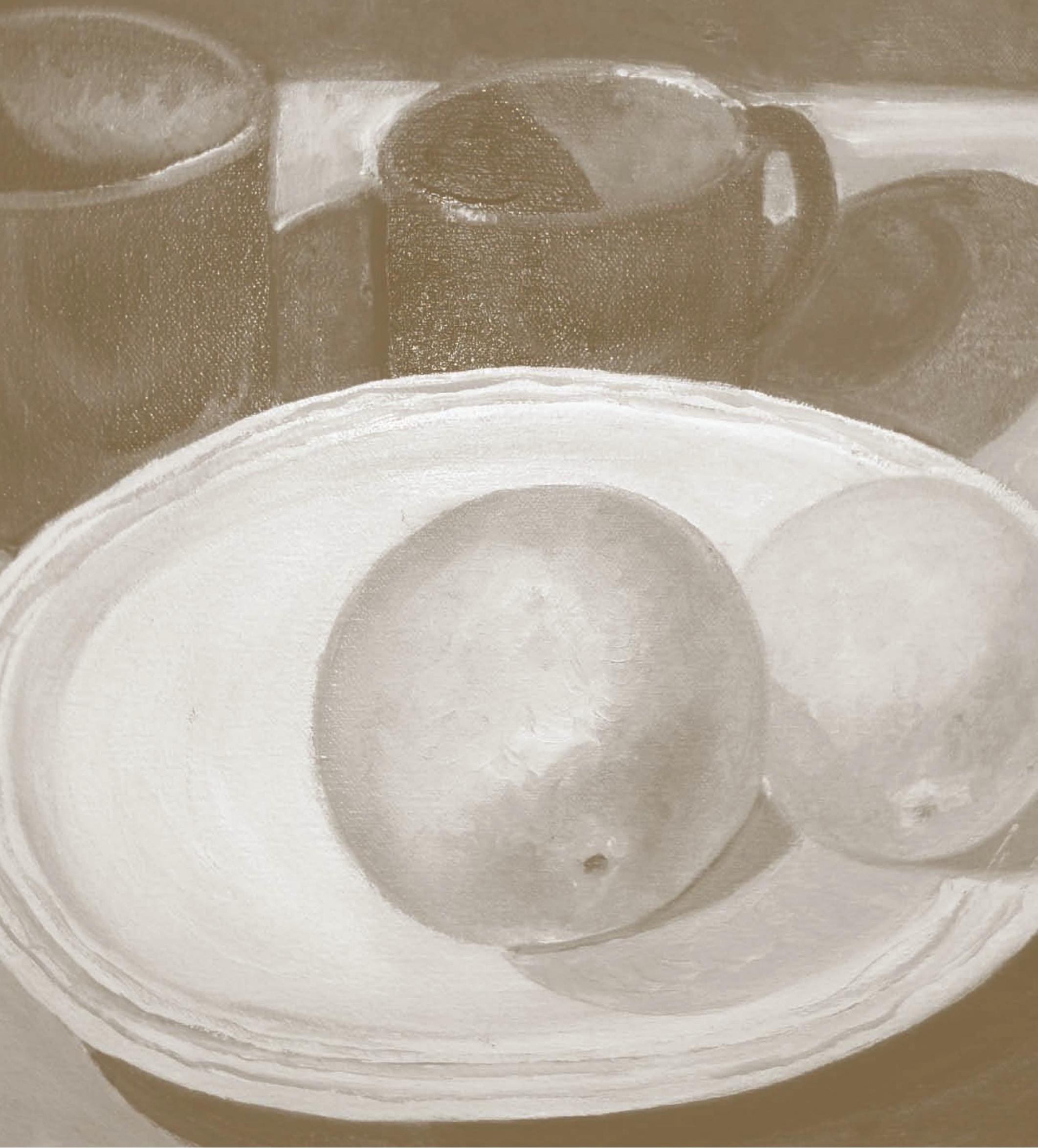




\section{Tendencias de la mortalidad en Honduras por grupos de edad, entre los años 1950-2005, con proyecciones al 2025}

HÉctor Yobany BACA

\section{INTRODUCCIÓN}

La mortalidad es una variable de la demografía y una de las principales responsabilidades de esta ciencia aplicada consiste en el estudio del crecimiento de la población y de sus componentes, para que sirva de base para la planificación económica y social.

En la presentación de este artículo se pretende dar a conocer las tendencias que ha tenido y tiene la mortalidad en Honduras y las que tendría si las condiciones de salud se mantienen igual.

Para entender estas tendencias se hacen señalamientos a algunas teorías de autores que se han interesado en el estudio de esta temática. Además se hace mención de algunos factores que inciden en estas tendencias.

Para determinar el grado de reducción o aumento de la mortalidad, se presentan algunos datos sobre la esperanza de vida al nacer de la población de Honduras con proyecciones al 2025. Las que permiten ver como las mujeres tienen una vida mas larga que los hombres.

\section{MORTALIDAD}

"La mortalidad es la desaparición permanente de toda evidencia de vida en cualquier momento después que ocurre el nacimiento vivo, o sea, cesación postneonatal de las funciones vitales, sin posibilidad de resucitación". ${ }^{1}$

La mortalidad es uno de los tres factores que junto con la fecundidad y la migración, determina los cambios que se producen en el tamaño, la distribución y la estruc- tura de la población. En la mayoría de los países, la mortalidad constituye el segundo factor de mayor importancia, después de la fecundidad en la determinación de estos cambios a escala nacional; a pesar de que la importancia de la migración aumenta cada vez más a medida que continúa la disgregación por debajo del nivel nacional. Las proyecciones demográficas son esenciales para toda planificación a largo plazo y también para gran parte de la planificación a plazos mediano y corto. ${ }^{2}$

Una particularidad de la mortalidad es que los riesgos de morir en los hombres es más elevado que el de las mujeres, este riesgo se mide por el exceso de mortalidad de los varones a través de la relación entre las tasas de hombres y de mujeres; a esta relación se le ha dado el nombre de índice de sobremortalidad masculina. ${ }^{3}$

Hernández, Raúl, ${ }^{4}$ plantea que de acuerdo a la experiencia internacional existen discrepancias notables en la mortalidad por sexo, incluso viviendo en las mismas condiciones ambientales generales. La mortalidad de las mujeres, en la mayor parte de los países del mundo, es menor a la de los hombres, a medida que ésta va disminuyendo, la brecha se va haciendo mayor. Estas diferencias se observan independientemente del nivel de desarrollo de los países. Si se tiene en cuenta la edad, también existe un diferencial favorable a las mujeres, pero ya no tan marcado como en la mortalidad general. Sin embargo, en la etapa anterior al nacimiento, los fetos masculinos reportan una mortalidad extremadamente superior a la femenina. La mayor mortalidad femenina en los primeros años de la infancia se ha atribuido a veces a la preferencia de los padres por los hijos varones y al consiguiente 
descuido de las niñas. La mortalidad por edades es menor para las mujeres en los países de baja mortalidad. No obstante, en algunos países subdesarrollados la mortalidad femenina es alta en algunos tramos de edad.

En la actualidad se pasa por un período de pesimismo general a uno de optimismo modesto y limitado, el estímulo proviene de avances reales en la comprensión de los procesos demográficos y la eficacia de estos para mejorar las proyecciones reales. ${ }^{5}$

José Cortés (1983), en su estudio sobre los determinantes socioeconómicos de la mortalidad en Colombia, centra el comportamiento de la mortalidad en dos grupos principales:

1. Factores asociados a un conjunto de servicios y actividades que influyen de manera directa en el estado de salud y mortalidad de la población (campañas preventivas y curativas).

2. Factores asociados al nivel de desarrollo social y económico del país, de los cuales depende la capacidad de acceso de las personas a la prestación de los diversos servicios de salud. ${ }^{6}$

Al determinar los tendencias de la mortalidad en Honduras, se hace un estudio de la transición demográfica, de la transición de la mortalidad lo que permite hacer un análisis comparativo con el resto de los países de la región y del mundo.

\section{La transición de la mortalidad}

La transición de la mortalidad, consiste en la reducción de la mortalidad, reflejado en el aumento de la esperanza de vida al nacer; esto posiblemente ha sido un logro en los países desarrollados, principalmente en las sociedades europeas.

Según el estudio de Vera Bolaños, ${ }^{7}$ se estima que algunas reducciones de la mortalidad podrían haber ocurrido en Europa occidental y son claramente identificables a fines del siglo XVIII y la primera mitad del siglo XIX.

Durante los estadios tempranos la elevación del ingreso contribuyó presumiblemente a la reducción de la mortalidad, aunque el desarrollo del estado moderno fue una influencia decisiva debido a la evolución de los servicios públicos tales como la provisión de agua potable, vacunación, control de epidemias y hambres, medidas de salud pública, la expansión de los períodos y áreas de paz en el estado moderno y en general al establecimiento de un orden público con el cual se disminuyó directamente el número de muertes ocasionadas por violencia.

Bolaños señala que el descenso inicial se debió esencialmente al avance tecnológico en la agricultura, el cual se reflejó en el mejoramiento en la nutrición y resistencia a enfermedades infecciosas, independientemente de la intervención médica y a las mejoras en la higiene, entre otras. Hay quienes atribuyen que la reducción en la mortalidad se debió a las mejoras en la higiene por el incremento en el consumo del jabón y de ropa lavable de algodón y la revolución en la medicina (siglo XIX), inducida por la formulación de la teoría infecciosa de la enfermedad, esta condujo a una reducción en la mortalidad en la niñez y más tarde en la mortalidad infantil. Posteriormente hubo grandes cambios en medicina, salud, educación y un gran número de adelantos tecnológicos y científicos que se observaron después de las dos guerras mundiales.

\section{ESTUDIO DE LA MORTALIDAD EN LOS ÚLTIMOS DIEZ AÑOS}

En el estudio de la mortalidad ha habido grandes cambios en las técnicas para extraer mediciones significativas de datos demográficos limitados y defectuosos. La mayoría de estos avances ha dependido de la construcción de modelos de los procesos demográficos, pero también han sido importantes otros como el de las verificaciones internas y el de la solidez de las relaciones. Estos avances en la metodología han sido acompañados por un mayor conocimiento y una comprensión mas profunda de la forma de las mediciones demográficas, por ejemplo, la composición por edades, del patrón de mortalidad, y de cómo estos cambian en el tiempo. ${ }^{8}$

Según la ONU (1985), ${ }^{9}$ históricamente y aún en la actualidad, la fuente de información más importante para el conocimiento de los niveles, tendencias y diferen- 
ciales de mortalidad ha sido el registro continuo de las defunciones y sus características en el registro civil. El propósito fundamental de esos sistemas ha sido usualmente administrativo, si bien su utilización marginal con fines estadísticos ha adquirido un nivel cada vez mayor al generalizarse el criterio de que el suministro de salud es una preocupación legítima de los gobiernos nacionales.

\section{MÉTODOS PARA LA MEDICIÓN DE LA MORTALI- DAD}

En la década de los ochenta se realizó la reunión del grupo de trabajo sobre: Base de Datos para la Medición de Niveles, Tendencias y Diferenciales de Mortalidad se convoco en Bangkok, Tailandia, del 20 al 23 de octubre de 1981. El objetivo de la reunión interregional fue debatir las experiencias de diversos órganos estatales e instituciones nacionales en la recopilación, análisis y utilización de datos de mortalidad a propósito para la formulación de políticas en los sectores de la salud y el desarrollo en sus países. ${ }^{10}$

Según la ONU (1985), existen numerosos métodos de recolectar datos para el estudio de la mortalidad, cada una de sus propias ventajas y desventajas en atención al costo, la precisión, la sencillez y la facilidad de vincularlo con otros registros Estos métodos se han utilizado en diversas combinaciones y en muchas versiones. En este trabajo se utiliza la siguiente clasificación sencilla de los métodos.

\section{A) Perspectivos:}

- Sistema de registro nacional, posiblemente sobre una muestra de las localidades.

- Registro de registro vitales y censos periódicos en un área especial de estudio.

B) Retrospectivos:

- Fichas ginecológicas.

- Informes sobre defunciones en el año anterior o en años anteriores;

- Métodos del tipo Brass, que utiliza datos sobre la sobrevivencia de los hijos, padres yo los cónyuges. ${ }^{11}$
Existen tres sistemas básicos para recoger datos sobre mortalidad a escala nacional:

- El sistema de registro civil.

- Las encuesta por muestreo

- Los censos de población.

Además se han utilizado algunas variantes de estos métodos básicos para la recopilación ya sea de datos nacionales o datos de mortalidad locales. ${ }^{12}$

El propósito fundamental de los estudios sobre mortalidad en Latinoamérica, el mundo, y principalmente en Honduras es el descubrimiento de los determinantes asociados con la mortalidad, y definir las medidas y programas para reducirla entre todos los grupos de la población. Los registros vitales, encuestas por muestreo y los censos nos proporcionan información directa sobre sus variables.

\section{ESPERANZA DE VIDA AL NACER DE LA POBLACIÓN DE HONDURAS ENTRE 1950-2025.}

Como resultado de algunas mejoras en las condiciones de la calidad vida de la población hondureña se ha logrado un aumento de 28.11 años en la esperanza de vida al nacer de los varones y 30.24 años en las mujeres,

\section{Gráfico No. 1. Tendencia de la esperanza de vida al nacer de la población de Honduras, 1950-2025}

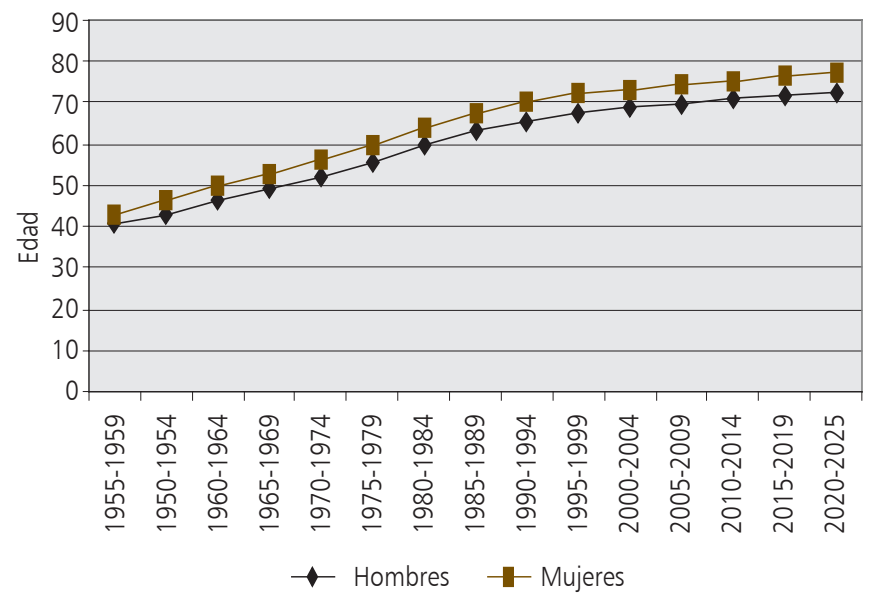

Fuente: Elaboración propia con datos de Naciones Unidas; CEPAL; CELADE; Boletín demográfico, América Latina: Tablas de mortalidad 1950-2025; año XXXIV, No. 67; Santiago de Chile; enero 2001. 
del año 1950 al 2005, y según proyecciones se espera un aumento de 3.89 años en los varones y 4.06 años en las mujeres del 2005 al 2025 si las condiciones se mantienen igual. ${ }^{13}$ Según el INE, 2004, la esperanza de vida al nacer de los hombres es de 68.4 años y para las mujeres es de 7.3 años.

Las mujeres tienen una esperanza de vida mas larga que los varones, esto posiblemente por el mayor riesgo de muerte a que son expuestos los hombres por causas como accidentes de transito, alcoholismo, drogadicción, labores fuera de la casas, etc.

La esperanza de vida al nacer es una variable que nos permite determinar si la mortalidad se ha reducido o aumentado. Al analizar la esperanza de vida al nacer de la población hondureña, en ambos sexos, se puede plantear que esta ha aumentado en 29.17 años de 1950 al 2005 y se espera que para el 2025 la esperanza de vida al nacer aumente 3.98 años.

En los años recientes el crecimiento de la población se ha desacelerado ligeramente, como consecuencia de la reducción gradual de la fecundidad, pero también se han mejorado las condiciones de salud; alargando más la esperanza de vida de la población. ${ }^{14}$ En la actualidad la esperanza de vida al nacer de los hombres es de 68.4 años, y la de las mujeres es de 75.3 años. ${ }^{15}$

\section{TENDENCIAS DE LA MORTALIDAD EN HONDURAS} ENTRE 1950-2025.

\section{Mortalidad de la población de 0-4 años}

$\mathrm{Al}$ analizar la mortalidad ${ }^{16}$ de la población de Honduras, entre las edades de 0-4 años, tanto para hombres y mujeres, se observa que esta ha tenido una tendencia decreciente a través del tiempo. Según el INE, 2004, la población en este grupo de edad es de 1,040,206 la cual equivale al $15.16 \%$ de la población total.

En el gráfico se observa que en 1950, la mortalidad en este grupo de población para ambos sexos mantenía niveles bajos, no obstante, posteriormente subió, alcanzando su máximo nivel en la segunda mitad de la década de los cincuenta.

\section{Gráfico No. 2. Mortalidad de la población de 0-4 años de edad, en Honduras, entre 1950-2025}

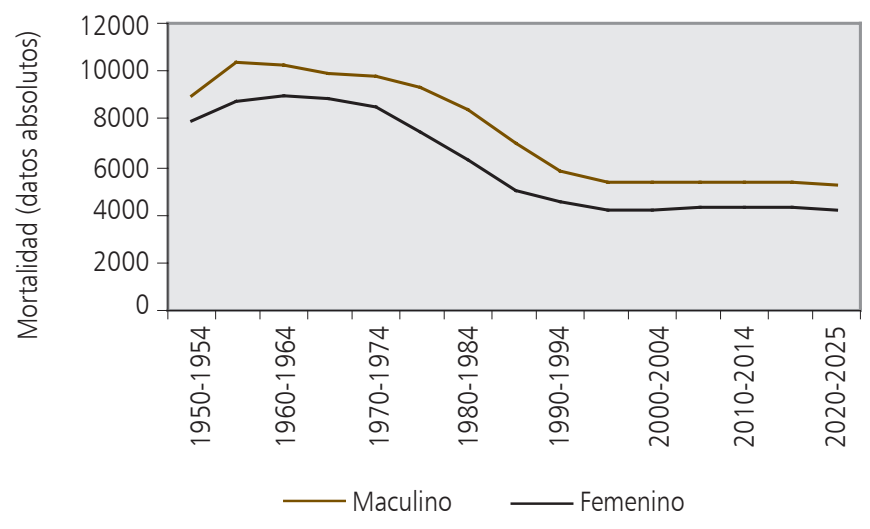

Fuente: Elaboración propia con datos de Naciones Unidas; CEPAL; CELADE; Boletín demográfico, América Latina: Tablas de mortalidad 1950-2025; año XXXIV, No. 67; Santiago de Chile; enero 2001.

La mortalidad masculina tiende a descender en este mismo periodo, en cambio la mortalidad femenina mantiene su nivel estable hasta la segunda mitad de la de la década de los sesenta. La mortalidad en ambos sexos desciende hasta inicios del nuevo siglo (XXI).

Del año 2000 al 2005 la mortalidad es estable tanto para hombres como para mujeres. Si las condiciones de salud se mantienen igual, se proyecta que la mortalidad seguirá teniendo la misma tendencia hasta el 2025 en ambos sexos. Una observación relevante de esta gráfica es que siempre se mantiene una sobremortalidad masculina en todo el periodo de estudio.

Uno de los mayores logros de salud pública es el descenso sostenido de la mortalidad infantil, especialmente a partir de 1930. La mortalidad infantil en Honduras a partir de esta década inicia su decrecimiento de una manera gradual, pero la caída se acelera a partir de 1940 a 1980, al bajar de 194 por 1000 a 65 por 1000 niños nacidos vivos. ${ }^{17}$ En la actualidad la tasa de mortalidad infantil estimada en hombres es de 34.6 y en las mujeres es de 26.8 por mil nacidos vivos, no obstante, la tasa de mortalidad de los niños de $1-4$ años es de 11 por mil nacidos vivos. ${ }^{18}$

La tendencia decreciente en los menores de cinco años, se refleja en el descenso de la mortalidad post neonatal, la cual baja de 17 a 15 muertes por 1000 naci- 
dos vivos entre los periodos de 1991-1995 y 1996-2000. en cambio entre los años 1986-1990 la mortalidad en la niñez se ha reducido de 16 a 11 muertes por 1000 nacidos vivos. ${ }^{19}$

A pesar de la reducción en la mortalidad infantil, siempre se mantienen niveles altos, esto se debe a que esta, tiene una relación directa con condiciones inadecuadas de acceso geográfico y social con la calidad de atención en los servicios. Un problema que provoca la muerte es el bajo acceso que tienen los niños a los servicios de salud y los que acceden tienen problemas de evaluación por parte del personal de los servicios. ${ }^{20}$

\section{MORTALIDAD DE LA POBLACIÓN DE 5-49 AÑOS DE EDAD}

Al analizar la mortalidad de la población de 5-49 años de edad, en ambos sexos, se observa que esta, tuvo una tendencia estable hasta el primer quinquenio de la década de los setenta. A partir de este periodo la mortalidad masculina tuvo un pequeña ascendencia en su tendencia de muertes el cual descendió al finalizar la primera mitad de la década de los ochenta, posteriormente esta tendencia aumento fuertemente.

En cambio la mortalidad femenina tuvo un pequeño descenso al iniciar la segunda mitad de los setenta este terminó hasta la primera mitad de los ochenta, luego ascendió fuertemente al igual que la mortalidad masculina.

Estas tendencias seguirán aumentando hasta el 2025, si se mantienen las mismas condiciones de calidad de vida de la población. En esta gráfica se puede observar que siempre se mantiene una sobremortalidad masculina en todo el periodo de estudio.

\section{MORTALIDAD DE LA POBLACIÓN MAYOR DE 50} AÑOS DE EDAD

Analizando la mortalidad de la población hondureña, mayor de 50 años de edad, tanto para hombres y mujeres, se observa que esta ha tenido una tendencia fuertemente ascendente a partir de la segunda mitad de la década de los setenta.

En el gráfico se observa que la mortalidad masculina tuvo una tendencia ascendente hasta el segundo quinquenio de los cincuenta, posteriormente descendió hasta la primera mitad de los setenta. A partir de este periodo la tendencia aumento sin interrupción.

En cambio la mortalidad femenina mantuvo un nivel estable hasta la segunda mitad de los cincuenta, a partir de este periodo empezó a descender hasta el primer quinquenio de los sesenta, luego tuvo un pequeño ascenso hasta la primera mitad de los setenta de aquí empezó de nuevo a descender hasta la segunda mitad de los setenta, desde entonces empezó a ascender fuertemente al igual que la mortalidad masculina.

La mortalidad en este grupo de población en la mayor parte del periodo de estudio a mantenido un nivel de crecimiento hasta la actualidad, lo que indica que la mortalidad para este grupo de población ha sido fuerte. Esto es consecuencia de la poca importancia que los gobiernos le dan a este grupo de población en la atención especial que se les debe brindar.

Estas tendencias seguirán aumentando hasta el 2025, si se mantienen las mismas condiciones de calidad de vida

\section{Gráfico No. 3. Mortalidad de la población de 5-49 años de edad en Honduras, entre 1950-2025}

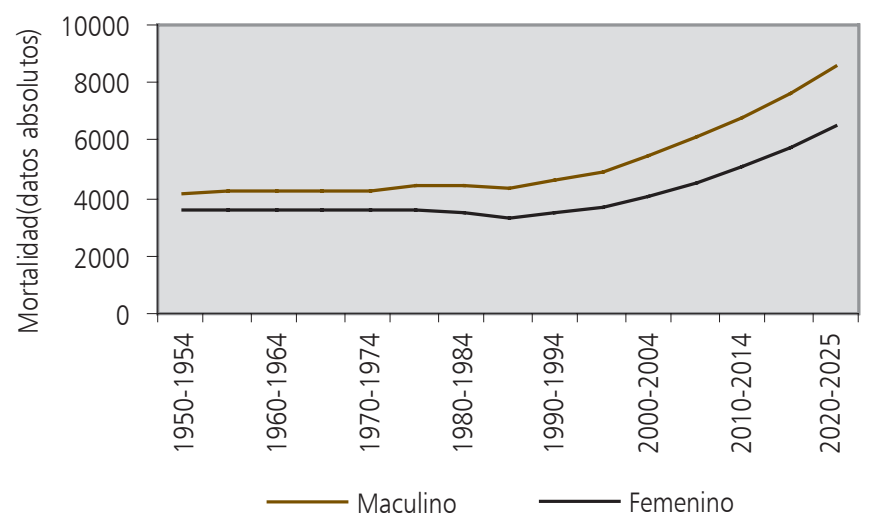

Fuente: Elaboración propia con datos de Naciones Unidas; CEPAL; CELADE; Boletín demográfico, América Latina: Tablas de mortalidad 1950-2025; año XXXIV, No. 67; Santiago de Chile; enero 2001. 
de la población. En esta gráfica se puede observar una diferencia de las otras dos anteriores; la sobremortalidad masculina no se mantiene en todo el periodo de estudio, hay un cambio en el quinquenio de 2015-2020 en el que se presenta una sobremortalidad femenina para este grupo de edad.

\section{Gráfico No. 4. Mortalidad de la población mayor de} 50 años de edad, en Honduras, entre 1950-2025

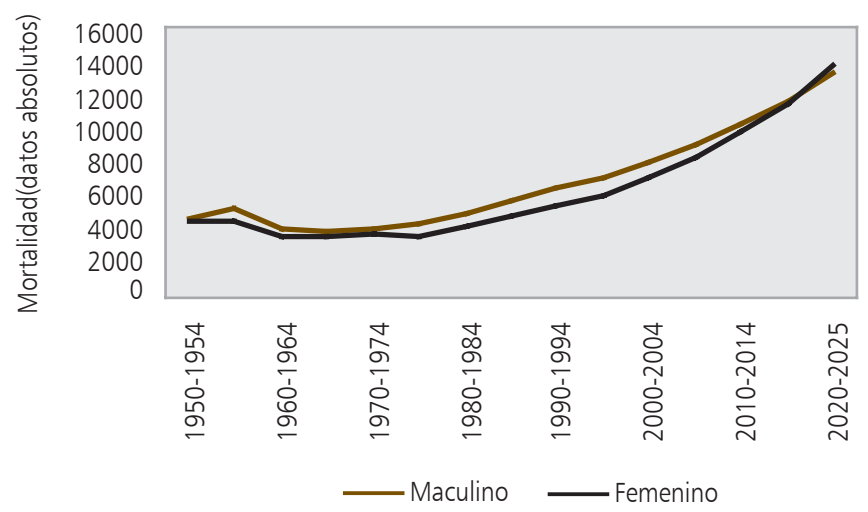

Fuente: Elaboración propia con datos de Naciones Unidas; CEPAL; CELADE; Boletín demográfico, América Latina: Tablas de mortalidad 1950-2025; año XXXIV, No. 67; Santiago de Chile; enero 2001.

\section{Inversión en programas de salud en Honduras}

Las tendencias de mortalidad en Honduras están determinadas por una serie de factores, entre estos se encuentran: atención médica hospitalaria, saneamiento ambiental, control de enfermedades transmisibles. Estos factores inciden en la mortalidad porque no brindan el total de sus servicios debido al bajo presupuesto con que cuentan para sus actividades. La inversión presupuestaria que se le asigna a cada uno de estos factores se detalla a continuación.

\section{ATENCIÓN MÉDICA HOSPITALARIA}

El presupuesto asignado a la atención médica hospitalaria no es un valor constante, este cambia de año con año. En el gráfico se observa la inversión que la Secretaria de Salud ha destinado en este programa del año 1986 al 2003. Se toman estas fechas porque es en este rango de años que se analizan las causas de muerte de la población hondureña.

\section{Gráfico No. 5. Presupuesto en Atención médica hospitalaria en Honduras}

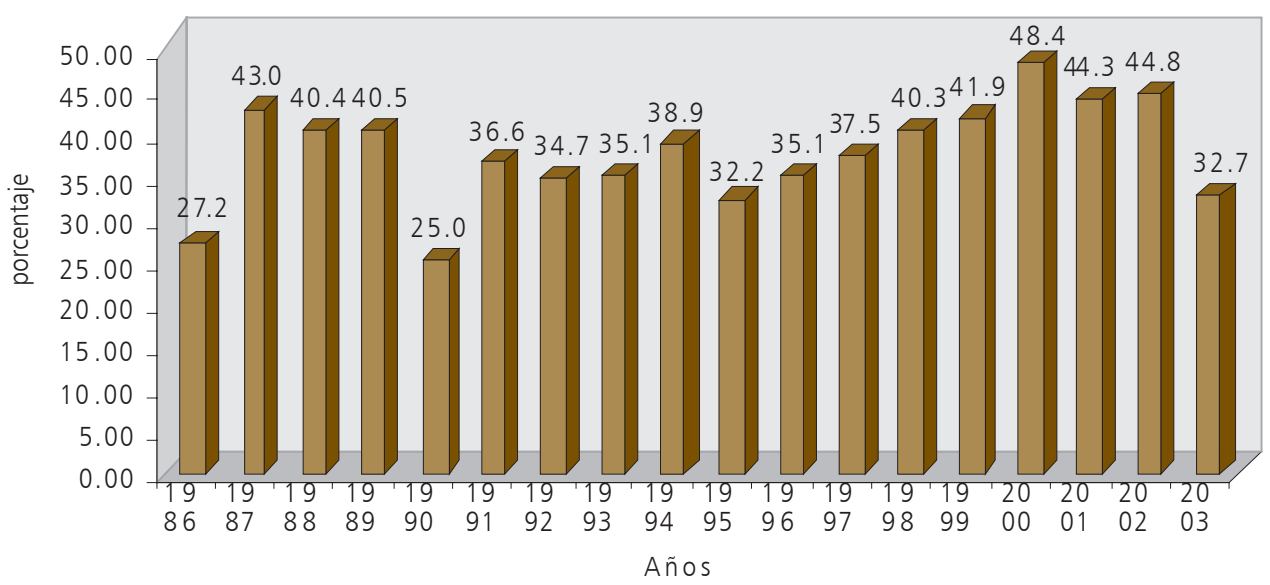

Fuente: Elaboración propia con datos de la Secretaria de Salud, 1986-1989, 1989-1993, 1994-1998, 1996-2000, 1997-2001 1999-2003; Salud en Cifras, Unidad de Planeamiento y Evaluación de la Gestión, Departamento de Estadística, Tegucigalpa M.D.C. (datos existentes) 


\section{SANEAMIENTO AMBIENTAL}

La vigilancia ambiental es uno de los puntos básicos en que la OPS apoya al Ministerio de Salud, priorizando lo que se refiere a fuentes de agua potable, aguas residuales domésticas e industriales, contaminación atmosférica, calidad de cuerpos receptores). En el aspecto de Atención Primaria Ambiental, se está trabajando con 3 comunidades y con escuelas primarias, creando clubes ecológicos ambientales para trabajar en pro del ambiente. Se cuenta con tecnología única en Latinoamérica, resaltando el uso de energía solar para la desinfección de desechos hospitalarios y los biofiltros, que permiten la reutilización del agua, en sectores con escasez del vital líquido. ${ }^{21}$
La inversión en saneamiento ambiental por parte de la Secretaria de Salud es mucho más reducido que el asignado a la atención médica hospitalaria.

De los años analizados se puede notar que el año, en el que se ha invertido más en este sector fue en 1988(7.09\%), y en 1989 (6.76\% del presupuesto total de esta secretaría).

Los años en los que menos se ha invertido en este rubro fue en el 2003 (3.82\%) y 2002 (4.41\%) respectivamente. Una observación importante de esto es que la inversión por parte de la secretaria de salud a este programa se ha reducido fuertemente en los dos últimos años en estudio $(2002,2003)$.

\section{Gráfico No. 6. Presupuesto en Saneamiento ambiental en Honduras}

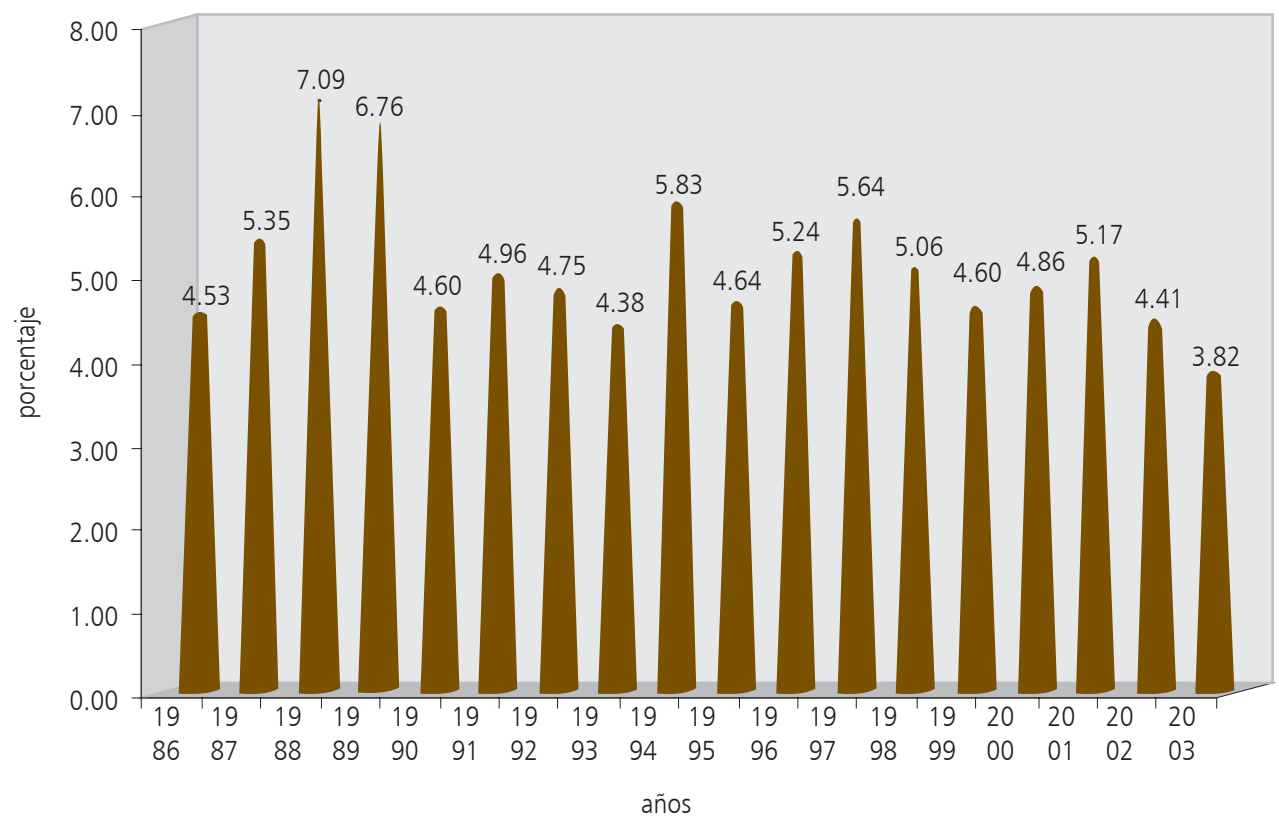

Fuente: Elaboración propia con datos de la Secretaría de Salud, 1986-1989, 1989-1993, 1994-1998, 1996-2000, 1997-2001, 1999-2003; Salud en Cifras, Unidad de Planeamiento y Evaluación de la Gestión, Departamento de Estadística, Tegucigalpa M.D.C. (datos existentes) 


\section{CONTROL DE ENFERMEDADES TRANSMISIBLES}

La inversión presupuestaria para el control de enfermedades transmisibles por parte de la Secretaría de Salud es mucho más reducida en los años 80 que en los años subsiguientes.

De los años analizados se puede notar que la menor inversión en este se dio en 1993(14.25\%), y en 1992 (15.81\% del presupuesto de esta secretaria).

Los años en los que más se ha invertido en este rubro fueron el 2001 (35.87\%) y 1997 (32.97\%) respectivamente. Una observación importante de esto es que la inversión por parte de la secretaria de salud a este programa se ha reducido en los dos últimos años en estudio (2002, 2003).

Las acciones coordinadas entre la Secretaria de Salud y la OPS, permitieron disminuir la incidencia de las enfermedades transmisibles, con balance final positivo a nivel nacional.
Los casos de dengue clásico se redujeron de más de 28,000 a 17,800, es decir una reducción del 33 por ciento. Los casos de dengue hemorrágico totalizaron 68, cifra únicamente comparable a la de 1998 con un total de 74 casos. Los casos de cólera se redujeron significativamente a menos de 60 en todo el año de 1999, cifra comparable a la situación de Honduras en 1992.

Para 1999 la situación de las diarreas en Honduras fue menor a la de 1998, año en que se registraron un total de 242,000 casos (209,000 en menores de 15 años), en tanto que en 1999 , las diarreas totalizaron 235,000 casos (entre ellos 188,000 en menores de 15 años); es decir una reducción nacional del 3 por ciento, pero con un significativo 10 por ciento en reducción de la enfermedad en menores de 15 años. ${ }^{22}$

$\mathrm{Al}$ analizar las primeras diez muertes que ocurren en Honduras por causa de enfermedad de mayor incidencia se observa que las enfermedades no transmisibles atacan mayormente a la población.

\section{Gráfico No. 7. Presupuesto en Control de enfermedades transmisibles en Honduras}

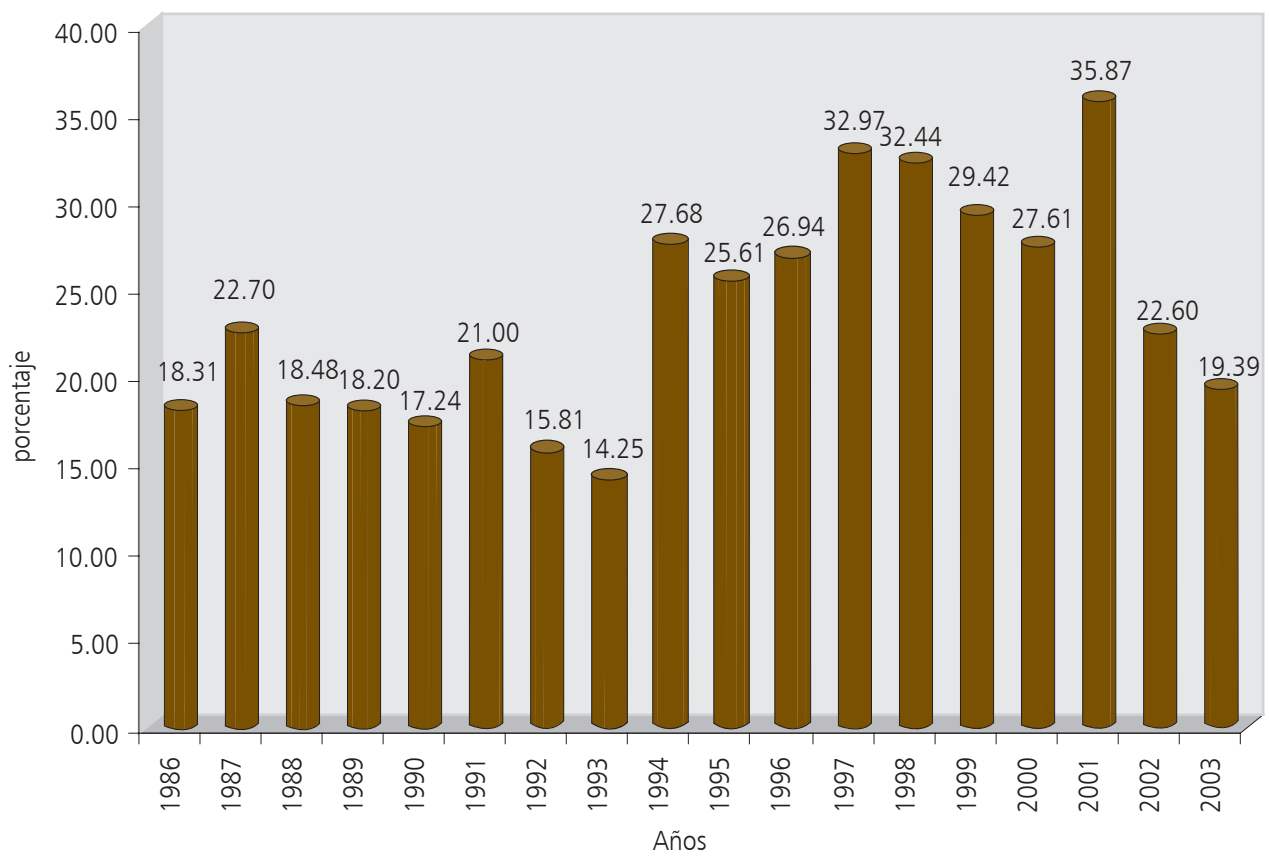

Fuente: Elaboración propia con datos de la Secretaría de Salud, 1986-1989, 1989-1993, 1994-1998, 1996-2000, 1997-2001, 1999-2003; Salud en Cifras, Unidad de Planeamiento y Evaluación de la Gestión, Departamento de Estadística, Tegucigalpa M.D.C. (datos existentes) 
La muerte por enfermedad transmisible que más incide en la población en los años analizados, es El SIDA y las diarreas de origen infeccioso.

La enfermedad no transmisible de mayor incidencia es el Síndrome de dificultad respiratoria y los accidentes no especificados esto nos revela que la influencia de contaminación ambiental y la falta de precaución en el manejo de automóviles por parte de los conductores, afectan fuertemente el nivel de crecimiento de toda la población en general.

Gráfico No. 8. Causas de muerte por enfermedades transmisibles y no transmisibles de mayor incidencia en cada año (1986-2003)

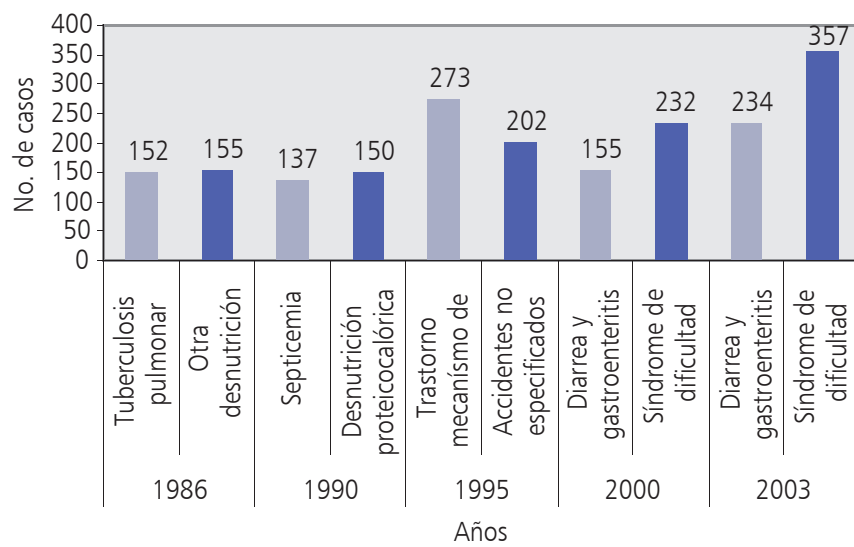

Fuente: elaboración propia con datos de la Secretaría de Salud de la República de Honduras, Boletín de Estadísticas Hospitalarias del año 1986 hasta el 2003, Dirección de Planeamiento y Evaluación de la Gestión Departamento de Estadística, Tegucigalpa MDC. (datos existentes).

\section{CONCLUSIONES}

- La mortalidad en Honduras, según los grupos de edad, presenta características diferenciadas en sus tendencias. La mortalidad para el grupo de 0-4 años tiene una tendencia contraria respecto a los grupos de 5-49 años y de 50 y más. Este grupo de 0-4 años tiene una tendencia decreciente de muertes y los otros dos grupos presentan una tendencia ascendente. No obstante la tendencia de muerte que presenta el grupo de 5 a 49 años es menos ascendente que la de los mayores de 50 años y más. Esto nos señala que hay mayor número de muertes en este último grupo, posiblemente por las condiciones inadecuadas de cuidado que requiere este grupo poblacional.

- En el análisis de las tendencias de la mortalidad se observa en todos los grupos una sobremortalidad masculina, sin embargo según proyecciones si las condiciones de salud se mantienen igual, se observa por la década de 2010 un pequeño cambio en la mortalidad de las personas de 50 años y más, en la que se presenta una sobremortalidad femenina. En el resto de los grupos ( $0-4$ y $5-49$ años respectivamente)se mantiene un sobremortalidad masculina. Esto se explica porque biológicamente los fetos y recién nacidos masculinos son más vulnerables ante las enfermedades.

- Las mujeres tienen una esperanza de vida al nacer mas larga que los varones, esto se debe en parte a que las mujeres son mas resistentes a algunas enfermedades en las primeras edades y también se debe a que los hombres son mas expuestos a causas como accidentes de transito, alcoholismo, drogadicción, etc.

- Para que las tasas de mortalidad continúen descendiendo se requiere que la inversión social sea sostenida y focalizada prioritariamente hacia los grupos poblacionales más vulnerables y que se ubican en zonas geográficas mas desprovistas de servicios básicos.

- De los años analizados se puede notar que los años en los que se ha invertido mas en atención médica hospitalaria fue en el 2000 y en el 2002. Sin embargo, produciendo una reducción en el 2003.

- La inversión presupuestaria para el control de enfermedades transmisibles por parte de la Secretaría de Salud fue mucho más reducida en los años 80 que en los años subsiguientes. 
CITAS

1. Hernández, Raúl; Septiembre, 2004; interpretación y análisis de datos demográficos I, Notas de Clase; UNAH, PLATS, Maestría en Demografía Social.

2. Naciones Unidas; 1985 ; base de datos para la medición de la mortalidad, Pág. 17.

3. Herrera, Lorenzo; 2005; Notas de Clase; UNAH, PLATS, Maestría en Demografía Social. Notas de clase, clase 3, tema 2: la mortalidad.

4. Hernández, Raúl; 2004; Notas de clase; Dinámica Demográfica I; Pág. 34; "Op. Cit."

5. Brass, William;1974; CELADE; Métodos para estimar la fecundidad y la mortalidad en poblaciones con datos limitados; Serie E, No.14; Santiago de Chile, 1974; Pág. 89..

6. Valladares, Lourdes; 2003; determinantes Socioeconómicos de la mortalidad en Honduras 1957-1983 (tesis); UNAH, PLATS; diciembre; 2003; Pág. 5

7. Vera Bolaños, Marta G., La teoría de la transición epidemiológica; "Op Cit"

8. Brass, William; 1974; Pág. 111; “op. ci.t.”.

9. Naciones Unidas; 1985; Pág. 2; "op. Cit."

10. Naciones Unidas; 1985; Pág. 7; "op. Cit.”

11. Naciones Unidas; 1985; Pág. 26; "op. Cit.”

12. Naciones Unidas; 1985; Pág. 37; "op. Cit.”

13. Como resultado de los progresos alcanzados en la reducción de la mortalidad y particularmente de la infantil, la esperanza de vida al nacer para ambos sexos aumenta moderadamente hasta 1950, al pasar de 34.0 años en 1930 a 41.9 en 1950, año en el cual inicia un rápido aumento, en concordancia con la baja en la tasa de mortalidad en el mismo período, pasando de los 41.9 años en 1950 a 61.6 en 1980, una ganancia de casi 20 años en tan solo 30 años. Posteriormente el aumento ha continuado, pero a un ritmo menor y la esperanza de vida se estima en 69.4 años en el periodo de 1995-2000. Banguero, 2000, Pág. 28

14. Banguero, 2000, Pág. 17

15. INE; 2004; indicadores basicos.

16. Tasa especifica de mortalidad conocida también como tasa central de mortalidad, se define como la relación entre las defunciones ocurridas en la población de determinada edad, o grupo de edades, y la población media del año.

17. Menjivar, Gladis; 2004; la mortalidad en la niñez en 16 municipios del corredor turístico centro sur de Honduras.

18. INE; 2004; indicadores básicos.

19. Menjivar, Gladis; 2004; "op, cit"

20. Secretaría de Salud Publica; 2002; Plan Estratégico Prioritario de Mortalidad infantil 2002-2006; Honduras.

21. Boletín Salud al Día - 1999, "Op Cit"

22. Boletín Salud al Día - 1999, "Op Cit”.

\section{BIBLIOGRAFÍA}

1. Banguero, Harold; 2000; Transición demográfica en Honduras; UNAH, PLATS; Tegucigalpa, Honduras.

2. Boletín Salud al Dia ; 1999 ; año 7, No. 17 www.paho-who. $\underline{\text { hn/resultad.htm }}$

3. Brass, William; 1974; CELADE; Métodos para estimar la fecundidad y la mortalidad en poblaciones con datos limitados; Serie E, No.14; Santiago de Chile.

4. Hernández, Raúl; septiembre, 2004; Dinámica demográfica I, Notas de Clase; Universidad Nacional Autónoma de Honduras(UNAH, Prostgrado Latinoamericano en Trabajo Social (PLATS), Maestría en Demografía Social (MDS).

5. Hernández, Raúl; septiembre, 2004; Interpretación y análisis de datos Demográficos I; notas de clase, Universidad Nacional Autónoma de Honduras (UNAH); Prostgrado Latinoamericano en Trabajo Social (PLATS); Maestría en Demografía Social (MDS).

6. Herrera, Lorenzo; 2005; notas de clase, tema 2, la mortalidad.

7. Menjivar, Gladis; 2004; la mortalidad en la niñez en 16 municipios del corredor turístico centro sur de Honduras; tesis de maestría; UNAH ;PLATS; MDS

8. ONU, 1985; Base de datos para la medición de la mortalidad; Nueva York; ST/ESA/SER.A184.

9. Secretaria de Salud de la República de Honduras; 1985 - 2002; Boletín de Estadísticas Hospitalarias; Dirección de Planeamiento y Evaluación de la Gestión Departamento de Estadística; Tegucigalpa, MDC.

10. Valladares, Lourdes; diciembre 2003; determinantes Socioeconómicos de la mortalidad en Honduras 1957-1983 (tesis); UNAH, PLATS, MDS.

11. Vera Bolaños, Marta G. *; Visión desde el punto de vista demográfico de la teoría de la transición epidemiológica postulada por Abdel Omran; http://www.cmq.edu.mx/docinvest/document/ DI39182.pdf 